David Chappell I University of West London, UK

\title{
CARDIOVASCULAR DISEASE: THE UNIVERSAL SLAYER
}

This voracious killer is strengthened by particular lifestyle choices

- can you escape its morbid embrace? 
ardiovascular disease is by far the leading cause of human mortality. Certain lifestyle choices lead to increased prevalence, however other forces are at play - over which we have no conscious control. The cardiovascular system comprises the heart and all of the blood vessels that are the conduit of flowing blood. It is a transport system providing oxygen and nutrients to respiring tissues, as well as removing waste products.

All human arteries comprise three main layers. The inner layer is made of cells called endothelial cells and the continuous lining created by these cells is called the endothelium. The endothelium is in direct contact with the flowing blood and is exquisitely sensitive to blood flow patterns. The main body of the artery wall is composed of protein fibres and smooth muscle cells. This part of the artery allows the vessel to expand or contract to regulate blood pressure. The endothelium and smooth muscle cells contain nervous tissue and are therefore responsive to messages from the nervous system. The outermost layer is composed of fibrous material, which allows it to be anchored within its environment.

It is known that dysfunction of the endothelium plays a role in the initiation of cardiovascular disease. Cardiovascular disease (CVD) occurs through the process of atherosclerosis which leads to the development of fatty deposits, called plaques, within the arterial wall (Figure $1 \mathrm{p} 40$ ). Plaque formation can result in reduced blood flow such that organ function can become compromised. The most common organs affected are the heart, leading to cardiac arrest, and the brain, increasing the likelihood of stroke.
Cardiovascular disease is a metabolic syndrome whereby individual risk factors can increase the likelihood of developing the disease. It is well known that certain lifestyle choices, for example a high calorie diet with little physical exercise, leading to obesity, contribute to CVD. However, other aspects are also involved, such as hypertension, diabetes and blood flow profiles. The multi-factorial nature of the disease is responsible for its staggering prevalence. According to the World Health Organisation, 17.9 million people died from CVD in 2016, which represented $31 \%$ of all global deaths. In the UK, $7.4 \mathrm{~m}$ people are living with CVD, and an average of 470 of these will die every day.

\section{Oxidative stress}

Oxidative stress is the key risk factor that can link the disparate elements associated with CVD. Oxidative processes occur naturally within the body through metabolism, and produce chemical species that are highly reactive. These reactive oxygen species (ROS), which include hydrogen peroxide and superoxide radicals, play essential roles within the body, including immune defence. However, excessive ROS can damage the tissues of the body through oxidative reactions, and normal levels are maintained through anti-oxidative measures. If these anti-oxidative mechanisms are insufficient to neutralise the effect of these processes, then a state of oxidative stress prevails. Oxidative stress exerts its negative effects on the body through a number of disease states. In addition to CVD, oxidative stress has also been implicated in other conditions including Parkinson's disease, Alzheimer's disease and cancer (Liguori et al, 2018). 


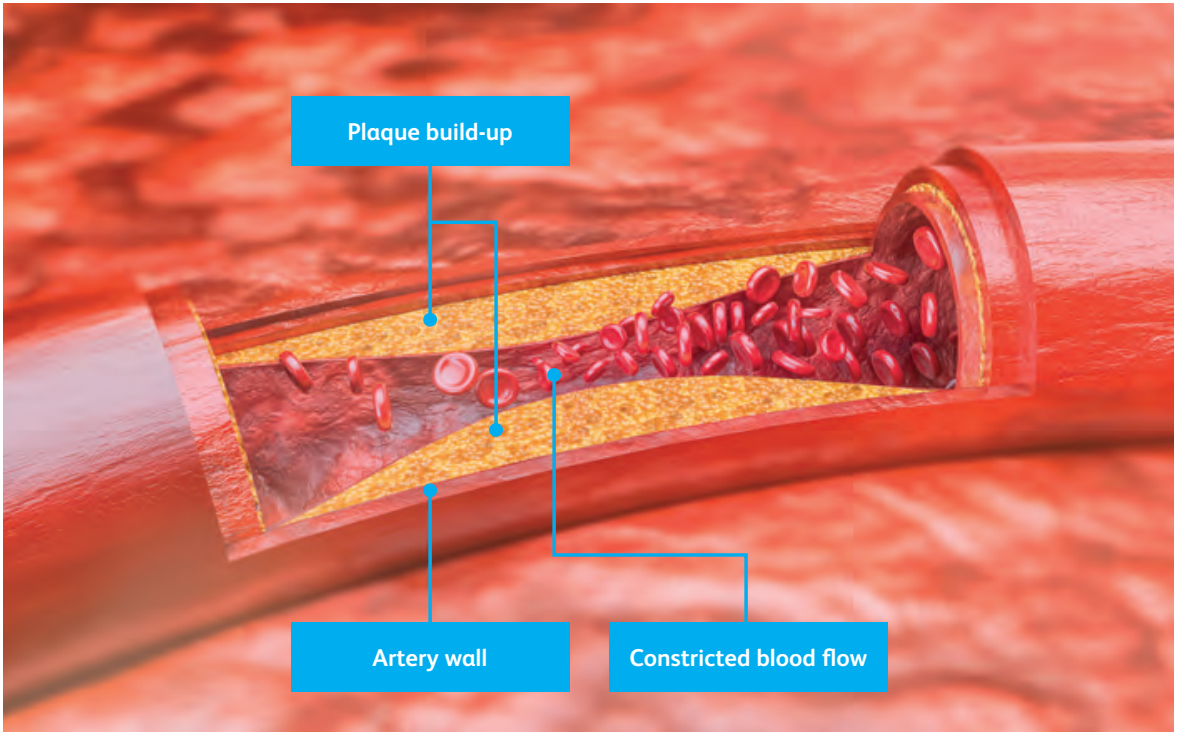

FIGURE 1 Arterial occlusion due to atherosclerotic plaque formation

\section{Atherosclerosis}

Cholesterol is an important component of al normal body cells. It is produced by the liver and is also derived from dietary sources. Cholesterol is not water-soluble and therefore needs to combine with protein to allow transportation within the blood stream. This cholesterol-protein complex is called a lipoprotein, and a number of lipoprotein variants exist. The type comprising a high protein to cholesterol ratio is called high-density lipoprotein $(\mathrm{HDL})$, whereas those with a low protein to cholesterol ratio are referred to as low-density lipoproteins (LDLS). It is these latter lipoproteins that play a fundamental role in the process of atherosclerosis. This is partly because the liver can only remove LDLs very slowly. As a consequence, LDLs travel within the blood for an extended period of time in relation to HDLs, and as LDLs are small they have both a greater opportunity and an increased ability to penetrate the arterial wall. LDLs gaining access to the arterial wall are readily oxidised by ROS to produce oxidised LDLs (oxLDLs). The creation of this oxidative product greatly accelerates the rate of atherosclerosis through a rapid development of atherosclerotic plaque.

\section{Obesity}

According to the World Health Organisation, an individual is said to be obese if their body mass index (BMI) is calculated to be 30 or above. BMI is derived from the weight in kilograms divided by the square of the height in metres. Obesity leads to disturbed lipid metabolism such that LDLs are broken down even less effectively, leading to increased circulating levels. To further compound the problem, obesity is able to raise oxidative stress within body cells (Lefranc et al, 2018) and the combination of LDLs and ROS ultimately increases levels of oxLDLs.

Biological sex can play a role as women are afforded some protection due to the fact that oestrogens are able to reduce ROS within body cells (Mahmoodzadeh \& Dworatzek, 2019). In addition, men tend to have higher blood LDL levels than women, and are therefore further disadvantaged with respect to CVD. In general, males and females have different hip to waist ratios. Moreover, the sites of body fat deposition vary according to sex whereby women tend to deposit body fat in areas such as the hips and thighs, whereas body fat distribution in men is more likely to be concentrated in the abdominal region (Kautzky-Willer, Harreiter \& Pacini, 2016).

\section{Diabetes mellitus}

Diabetes mellitus is a metabolic disorder whereby levels of a type of sugar (glucose) within the blood are not adequately controlled. Insulin is the hormone released from the pancreas that instructs cells of the body, for example the liver and skeletal muscle cells, to uptake glucose from the blood. Insulin is the only hormone produced by the body that is able to lower blood glucose concentrations. A number of hormones, for example adrenalin and growth hormone, are able to increase blood glucose levels, and it is the interplay between these hormones and insulin that normally maintains tight control of blood glucose levels.

There are two main types of diabetes mellitus (hereafter referred to as 'diabetes'), type 1 and type 2. Type 1 diabetes occurs as a result of impairment or destruction of the cells of the pancreas that are responsible for producing insulin and therefore insulin is either present in extremely low levels or is absent. The major feature of type 2 diabetes is insulin resistance. In this case, insulin is released from the pancreas, however the body cells do not receive a clear instruction to remove glucose from the blood. As a consequence, both types of diabetes result in abnormally elevated levels of blood glucose. Glucose is quite reactive and is able to chemically attach itself to proteins and this has implications for regulating blood pressure.
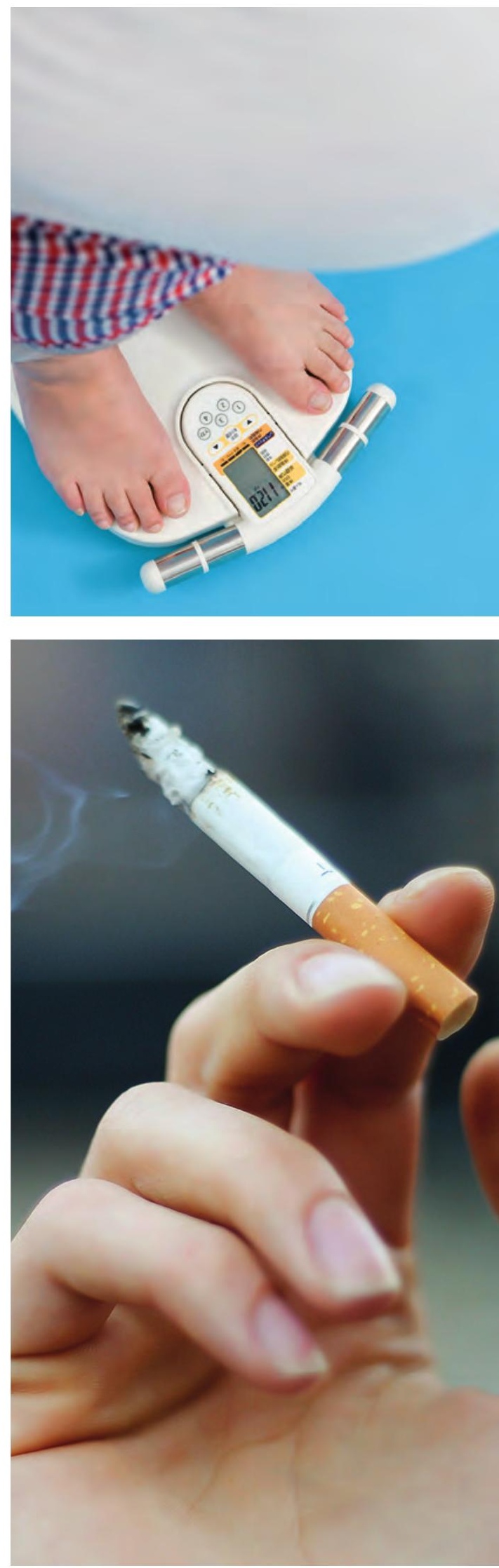


\section{The normal human heart contracts on average 60-90 times per minute, and generates a unidirectional, pulsatile blood flow profile. As arteries carry blood away from the heart, the blood is rapidly transported through these vessels}

system and lead to the production of ROS (Wu et al, 2018). Reactive oxygen species then chemically react with NO so that the levels of active NO are decreased even further (Liguori et al, 2018). This significant reduction in $\mathrm{NO}$ levels due to the effects of hypertension and ROS generated through exposure to nicotine and tobacco smoke, impairs the ability of blood vessels to expand, leading to chronic hypertension.

Diabetes (primarily type 2 ) is able to affect lipid metabolism. In this case, the ratio of LDLs to HDLs within the blood is skewed in favour of LDLs. The fact that LDLs remain in the blood stream for longer periods of time relative to HDLs, coupled with the knowledge that higher levels of LDLs are present as a consequence of diabetes type 2, highlights the clear link between diabetes and atherosclerosis. A primary cause of type 1 and type 2 diabetes has been attributed to oxidative stress through damage to the pancreatic cells that produce insulin (Ullah, Khan \& Khan, 2016).

\section{Hypertension and tobacco smoke}

Normal blood pressure is generally regarded as a systolic (heart contracted) blood pressure of $120 \mathrm{mmHg}$ and diastolic (heart relaxed) blood pressure of $80 \mathrm{mmHg}$. High blood pressure, or hypertension, is clinically defined as a systolic blood pressure greater than $140 \mathrm{mmHg}$ and/or diastolic blood pressure greater than $90 \mathrm{mmHg}$. Nitric oxide (NO) is a gas released by normal endothelium that is able to relax arterial smooth muscle cells. This relaxation leads to expansion of the blood vessel, which effectively reduces blood pressure. Obesity is able to stimulate nervous tissue present within the arterial wall, leading to continuous smooth muscle contraction which brings about a sustained increase in blood pressure. Prolonged hypertension can damage the arterial wall leading to endothelial dysfunction, with the effect of promoting atherosclerosis.

Tobacco smoke is made up of a wide variety of chemicals such as benzene and pyrenes, heavy metals such as nickel and lead, and toxic gases including carbon monoxide and ammonia. This chemical cocktail can exert profoundly negative effects on the lungs, liver and kidneys. The chemicals in tobacco smoke, in addition to nicotine, can also adversely affect the vascular

\section{Blood flow profiles}

The normal human heart contracts on average 60-90 times per minute, and generates a unidirectional, pulsatile blood flow profile. As arteries carry blood away from the heart, the blood is rapidly transported through these vessels. The blood is moved most quickly through the centre of the blood vessel, whereas the blood passing over the endothelium is slowed due to the frictional force (shear stress) generated by the blood flow across the endothelial surface. The endothelial cells respond to shear stress by elongating and lining up in the direction of flow, and this cellular arrangement tends to resist penetration of circulating LDLs. As a consequence, this flow profile which produces a high fluid shear stress, has the potential to retard atherosclerosis.

The initiation of atherosclerosis is highly focal in nature whereby branch points within the arterial system are the first to be affected. Vascular branch points divert the blood through each branch thereby slowing down the flow and decreasing the fluid shear stress. At certain points, the positive forward flow can be slowed to such a degree that there is backward flow also, and this results in the endothelium experiencing an oscillatory flow profile at these points. Endothelial cells exposed to oscillatory flow are not elongated or aligned, but assume a random cobblestone arrangement (Chappell et al, 1998). Oscillatory flow profiles have been found to activate an intracellular enzyme called NADH oxidase (De Keulenaer et al, 1998). Enzymes are proteins that are involved in metabolic processes, and NADH oxidase takes part in biochemical reactions that produce superoxide radicals. As a consequence, oscillatory shear stress has the potential to increase cellular oxidative stress by the production of ROS. As previously described, increased levels of superoxide radicals are likely to lead to greater abundance of oxLDLs resulting in increased levels of atherosclerotic plaque. 
Oxidative stress is the key factor in the initiation and development of atherosclerosis, and it is likely that individuals who are less effective in negating the deleterious effects of oxidative stress are more likely to suffer from CVD

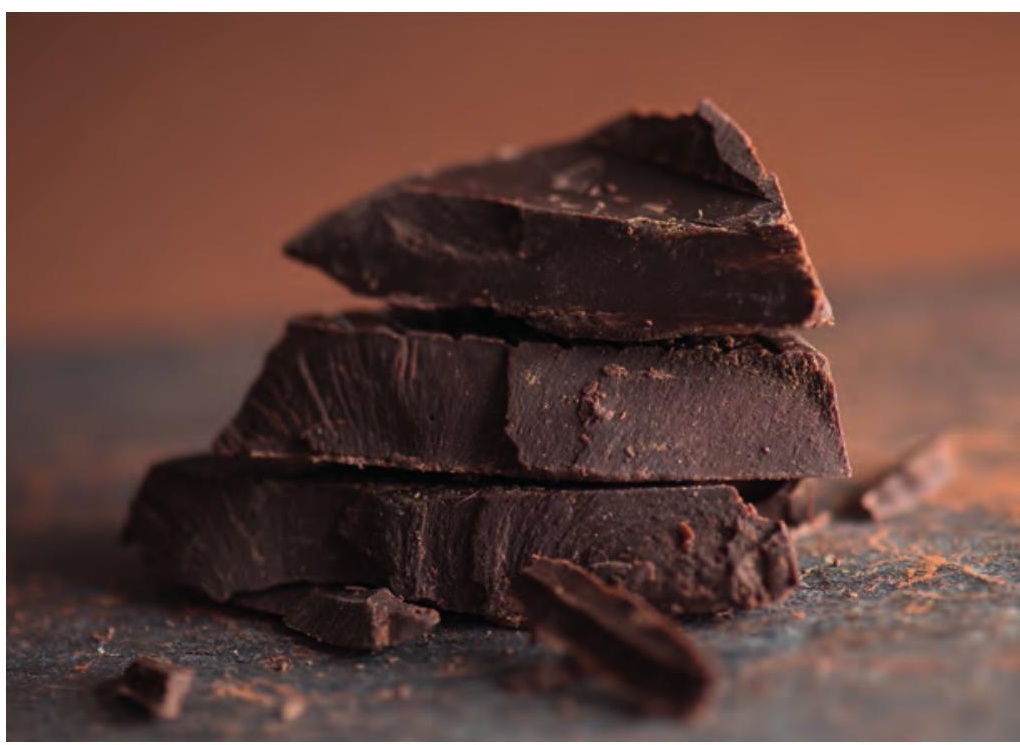

\section{Treatments and emerging therapies}

Conventional treatments for CVD include balloon angioplasty and coronary artery bypass surgery. Balloon angioplasty involves the insertion of a catheter into an artery, carrying a balloon and a stainless steel mesh (stent) at the tip. On reaching the narrowed blood vessel, the balloon is inflated so that the plaque is pushed to the sides of the blood vessel wall. The stent is then deployed maintaining the opened blood vessel. Coronary artery bypass surgery is performed when the affected artery is blocked, and involves using blood vessels from other parts of the body, for example the legs, to bypass the affected artery in order to increase blood supply to the heart. Medical interventions now tend to focus on preventative measures. These include the use of drugs to control hypertension, and one such set of medications are the ACE-inhibitors. These control the activity of an enzyme which has the ability to raise blood pressure. As a consequence, inhibition of this enzyme will naturally reduce hypertension. Drugs (statins) have also been deployed to reduce cholesterol (and therefore LDL) production by the liver, as well as promote the removal of circulating LDLs.

Emerging therapies are becoming more sophisticated by targeting specific mediators of atherosclerosis. For example, it has been found (Tang \& Hazen, 2017) that a particular enzyme (PCSK9), in combination with other therapies, is able to reduce the ability of the liver to take up LDLs from the circulation. It follows therefore that targeted inhibition of this enzyme, may have a therapeutic effect with regard to CVD, by allowing the liver to more easily remove LDLs from the circulation. Dysfunctional cells and debris within the plaque would normally be removed by scavenger cells (macrophages) - with the potential to reduce plaque size. However, these plaque cells through biological messages, are able to prevent macrophages from degrading the plaque (Tang \& Hazen, 2017). Blockade of this biological instruction could promote removal of plaque constituents by macrophages and thereby reduce plaque size, and possibly reverse the atherosclerotic process.

\section{Exercise and diet}

Regular moderate physical exercise, in conjunction with a calorie-controlled diet, will help to reduce excess body weight and has been suggested to promote anti-oxidative processes within cells so that ROS are decreased (Ligouri et al, 2018). This dual action would provide a range of additional benefits as reduced body fat would help to lower circulating LDLs, and as reactive oxygen species are decreased, there would be an overall decreased production of oxLDLs. Moreover, a reduction in abdominal fat (in males) would help to control or decrease the likelihood of developing type 2 diabetes. In addition, generally decreased body fat would lower blood pressure, thereby reducing damage to the endothelium, and promoting the release of NO.

Homocysteine is a product of metabolism that can increase oxidative stress, with the potential to promote atherosclerosis. Homocysteine is quickly and easily removed by a process which uses vitamin B12. However, the body is not able to produce this vitamin and dietary sources are restricted to products of animal origin. This could have implications for those following vegan diets. It has been shown (Obersby et al, 2015) that vegans, compared to omnivores, are statistically more likely to display raised blood homocysteine levels.

Dietary vigilance can also play an important role in preventing or slowing the development and progression of CVD. The effects of type 2 diabetes can be reduced by following an appropriate diet leading to gradual release of glucose (low glycaemic index). Certain foodstuffs are beneficial in that they are processed by the body to produce anti-oxidants that are able to neutralise ROS. These include blueberries, artichokes, tomatoes and dark chocolate. Vitamin C (found in citrus fruits, for example oranges) and vitamin $\mathrm{E}$ (sourced from foods such as avocados and tuna) are natural anti-oxidants. Herbs and spices such as ginger and turmeric, through their anti-oxidant nature, have also been shown to interfere with a number of atherosclerotic processes (Nimgulkar et al, 2015).

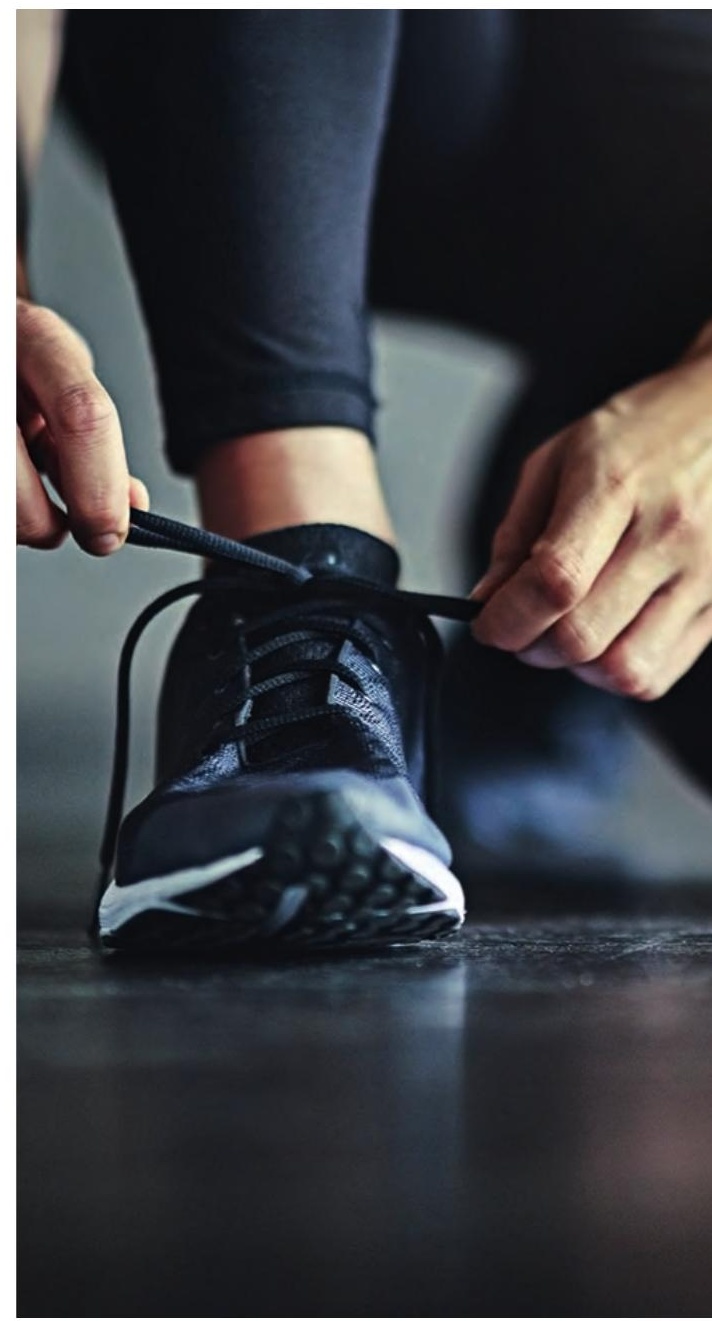




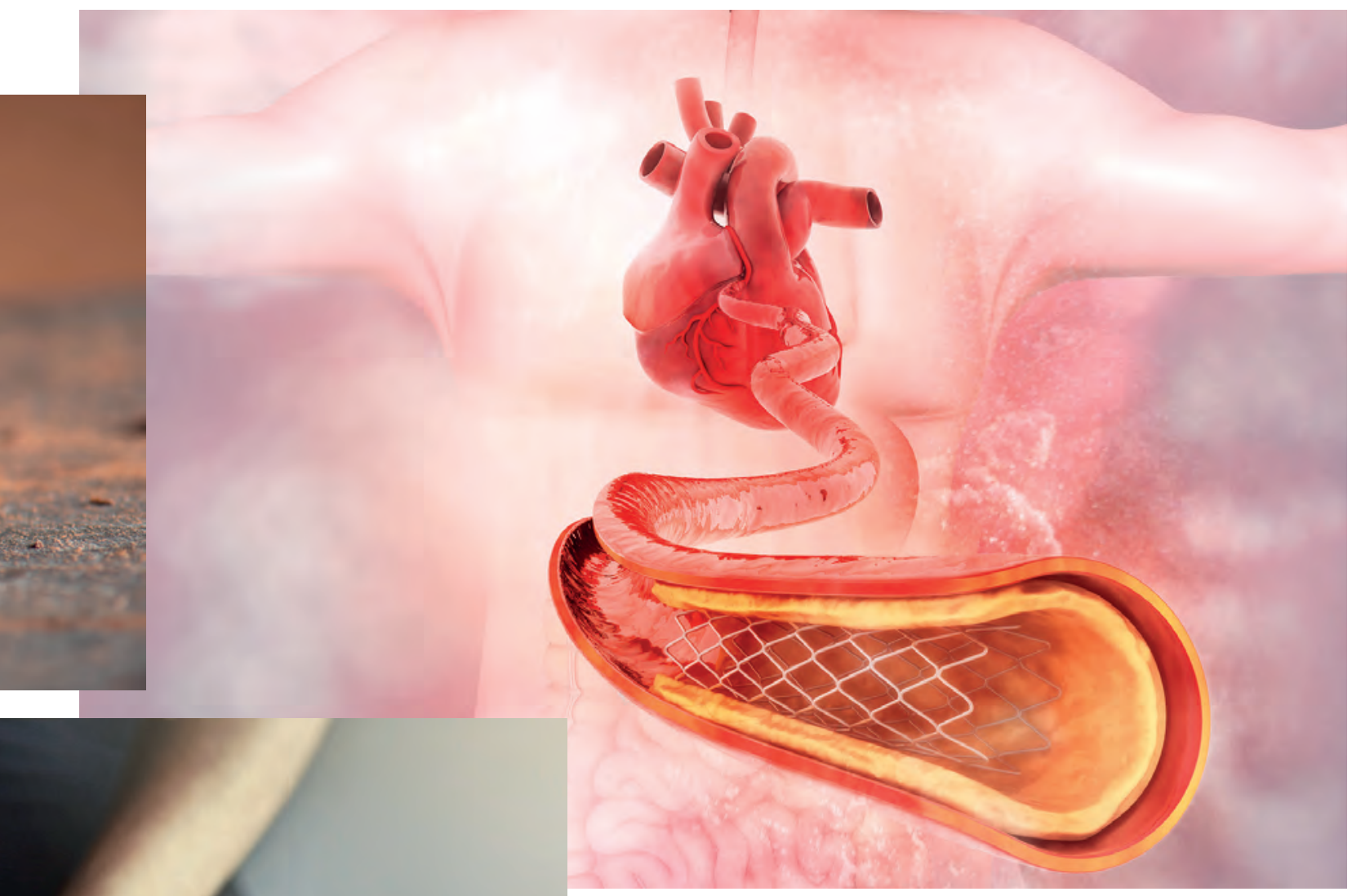

\section{References}

Chappell, D.C. et al (1998) Oscillatory shear stress stimulates adhesion molecule expression in cultured human endothelium. Circulation Research, 82: 532-539

De Keulenaer, G.W. et al (1998) Oscillatory and steady laminar shear stress differentially affect human endothelial redox state. Circulation Research, 82: 1094-1101 Kautzky-Willer, A., Harreiter, J. \& Pacini, G. (2016) Sex and gender differences in risk, pathophysiology and complications of type 2 diabetes mellitus. Endocrine complications of type 2 diat
Reviews, 37(3): 278-316

Lefranc, C. et al (2018) Mitochondrial oxidative stress in obesity: role of the mineralocorticoid receptor. Journal of Endocrinology, 238(3): R143-R159

Liguori, I. et al (2018) Oxidative stress, aging, and diseases. Clinical Interventions in Aging, 13: 757-772

\section{Conclusion}

A number of diverse factors play important roles in the development of CVD through atherosclerotic plaque formation. These include high blood cholesterol levels, smoking, diabetes mellitus, hypertension, blood flow profiles and biological sex. It is often the case that these elements combine to exacerbate the problem and/or promote the likelihood of disease. Oxidative stress is the key factor in the initiation and development of atherosclerosis, and it is likely that individuals who are less effective in negating the deleterious effects of oxidative stress are more likely to suffer from CVD. Considering its multi-factorial nature it is probable that CVD will remain the most likely cause of human mortality in the near future. However, therapies which aim to reduce oxidative stress, in combination with sensible lifestyle choices, including diet, have the potential to be more effective in preventing the development of CVD.

Mahmoodzadeh, S. \& Dworatzek, E. (2019) The Role of $17 \bigotimes$-estradiol and estrogen receptors in regulation of $\mathrm{Ca} 2+$ channels and mitochondrial function in cardiomyocytes. Frontiers in Endocrinology, 10: 310-313

Nimgulkar, C. et al (2015) Combination of spices and herbal extract restores macrophage foam cell migration and abrogates the athero-inflammatory signalling cascade of atherogenesis. Vascular Pharmacology, 72: 53-63

Obersby, D., Chappell, D., Dunnett, A. \& Tsiami, A. (2015) Efficacy of methylcobalamin to normalise elevated homocysteine of vitamin B12 deficient vegetarians: A double blind placebo control study. Current Research in Nutrition and Food Science 3: 187-196

Tang, W.H.W. \& Hazen, S.L. (2017) Advances in new therapeutic targets for atherosclerosis. Nature reviews. Cardiology. 14(2): 71-72

Ullah, A., Khan, A. \& Khan, I. (2016) Diabetes mellitus and oxidative stress - a concise review. Saudi Pharmaceutical Journal, 24(5): 547-553

Wu, X. et al (2018) Nicotine promotes atherosclerosis via ROSNLRP3-mediated endothelial cell pyroptosis. Cell Death and Disease, 9: 171-182

\section{About the authors}

Dr David Chappell, Senior Lecturer in Physiology, University of West London.

\section{Keywords}

Cardiovascular Disease, Atherosclerosis, Risk Factors,

Oxidative Stress 